\title{
An exploratory cohort study of sensory extinction in acute stroke: prevalence, risk factors, and time course
}

\author{
Joseph Kamtchum-Tatuene ${ }^{1,2}$ (ib $\cdot$ Gilles Allali $^{1,3} \cdot$ Arnaud Saj $^{1,4} \cdot$ Thérèse Bernati $^{1}$. \\ Roman Sztajzel $^{1} \cdot$ Pierre Pollak $^{1} \cdot$ Isabelle Momjian-Mayor $^{5} \cdot$ Andreas Kleinschmidt $^{1}$
}

Received: 3 October 2016/ Accepted: 1 December 2016/Published online: 9 December 2016

(c) The Author(s) 2016. This article is published with open access at Springerlink.com

\begin{abstract}
Most studies on sensory extinction have focused on selected patients with subacute and chronic right hemisphere lesions. In studies conducted on acute stroke patients, risk factors and time course were not evaluated. Our aim was to determine the prevalence, risk factors, and time course of sensory extinction in the acute stroke setting. Consecutive patients with acute stroke were tested for tactile, visual, auditory, and auditory-tactile cross-modal extinction, as well as for peripersonal visuospatial neglect $(\mathrm{PVN})$. Tests were repeated at 2, 7, 15, 30, and 90 days after initial examination. A multivariable logistic regression analysis was performed to test the association between sensory extinction and demographic and clinical risk factors. Seventy-three patients (38.4\% women) were recruited: 64 with ischemic stroke and nine with haemorrhagic stroke. Mean age was 62.3 years (95\% CI 58.8-65.7), mean
\end{abstract}

Electronic supplementary material The online version of this article (doi:10.1007/s00702-016-1663-x) contains supplementary material, which is available to authorized users.

Joseph Kamtchum-Tatuene

tatuene@liv.ac.uk

1 Neurology Division, Department of Clinical Neurosciences, Geneva University Hospital, Geneva, Switzerland

2 Brain Infections Group, Institute of Infection and Global Health, University of Liverpool, 8 West Derby Street, Liverpool L69 7BE, UK

3 Division of Cognitive and Motor Aging, Department of Neurology, Albert Einstein College of Medicine, Yeshiva University, Bronx, NY, USA

4 Neurology and Cognitive Imaging Laboratory, Faculty of Medicine, University of Geneva, Geneva, Switzerland

5 Neurology and Neurophysiology Centre, Geneva, Switzerland
NIHSS score was 1.6 (95\% CI 1.2-2.1), and mean time to first examination was 4.1 days (95\% CI 3.5-4.8). The overall prevalence of all subtypes of sensory extinction was $13.7 \%$ (95\% CI 6.8-23.8). Tactile extinction was the most frequent subtype with a prevalence of $8.2 \%$ (95\% CI 3.1-17.0). No extinction was found beyond 15 days after the first examination. After adjustment for age, sex, lesion side, type of stroke, time to first examination and stroke severity, a lesion volume $\geq 2 \mathrm{~mL}$ (adjusted $\mathrm{OR}=38.88$, $p=0.04$ ), and presence of PVN (adjusted $\mathrm{OR}=24.27$, $p=0.04$ ) were independent predictors of sensory extinction. The insula, the putamen, and the pallidum were the brain regions most frequently involved in patients with sensory extinction. Extinction is a rare and transient phenomenon in patients with minor stroke. The presence of $\mathrm{PVN}$ and lesion volume $\geq 2 \mathrm{~mL}$ are independent predictors of sensory extinction in acute stroke.

Keywords Acute stroke - Sensory extinction . Visuospatial neglect $\cdot$ Risk factors $\cdot$ Prognosis

\section{Introduction}

Extinction is a behavioural symptom of brain lesions where patients report, respond, or orient to a stimulus presented on the contralesional side, but fail to detect the same stimulus when presented concurrently with another stimulus on the ipsilesional side (Bender 1952; Chechlacz et al. 2014; de Haan et al. 2012). Extinction frequently co-occurs with visuospatial neglect and the question of whether they should be considered as common or separate deficits is still debated. Indeed, several authors still consider extinction as a milder form or a residual manifestation of neglect after recovery (Heilman and Valenstein 2012; Liu et al. 2011; 
Vuilleumier and Rafal 2000), while others support the idea that extinction and neglect are separate deficits (Karnath and Rorden 2012; Priftis et al. 2013; Vossel et al. 2011). The latter view relies on the fact that both syndromes can occur independently (Cocchini et al. 1999; Di Pellegrino and De Renzi 1995) and have frequently been related to distinct neuroanatomical substrates (Karnath et al. 2003; Karnath and Rorden 2012; Vallar et al. 1994).

Neglect can be sensory, visuospatial, motor, representational, or personal (Heilman et al. 2012; Punt et al. 2013; Saj and Vuilleumier 2013), and extinction can be motor or sensory. Sensory extinction can be further classified as unimodal (tactile, visual, or auditory), multimodal, or cross-modal (Jacobs et al. 2011). Unbalanced attentional competition between brain hemispheres (Driver and Vuilleumier 2001; Kinsbourne 1977; Riddoch et al. 2009) and impaired processing of contralesional sensory stimuli in the absence of a primary sensory deficit (Chechlacz et al. 2014; Rorden et al. 2009; Watling et al. 2013) are the mechanisms most frequently proposed to explain the occurrence of extinction. Such putative mechanisms can explain the classical extinction scenario where contralesional stimuli are extinguished in the context of double simultaneous stimulation, but recent descriptions of "antiextinction" (Humphreys et al. 2002; Watling et al. 2013) and "ipsilesional extinction" (de Haan et al. 2015) suggest more complex pathomechanisms also involving a nonspatial deficit of attentional capacity at least in some patients with extinction. Anti-extinction occurs when there is poor report of a single stimulus presented on the contralesional side of space, but better report of the same item when it occurs concurrently with a stimulus on the ipsilesional side (Humphreys et al. 2002). Ipsilesional sensory extinction refers to failure to report an ipsilesional stimulus when presented simultaneously with a contralesional stimulus, while there is normal reporting of single ipsi- and contralesional stimuli (de Haan et al. 2015; Karnath 1988). Moreover, recent demonstrations of sensory extinction in healthy individuals highlight the existence of multisensory integration neuronal networks whose impairment could lead to altered perception of stimuli from either side of space no matter on which side a brain lesion is located (Jacobs et al. 2011). Lesions of multimodal neurons could be involved in the pathophysiology of ipsilesional crossmodal extinction by inducing mislocalization or misidentification of stimuli (Liu et al. 2011).

Most studies on extinction have been conducted on selected patients with subacute and chronic brain lesions (Chechlacz et al. 2014; Vallar et al. 1994; Vuilleumier 2013). The rare studies conducted on patients with acute stroke did not evaluate risk factors and time course of extinction (Becker and Karnath 2007) and some only reported patients with right hemisphere lesions (Umarova et al. 2011; Vallar et al. 1994; Vossel et al. 2011). Our aim here was, therefore, to gather data on prevalence, risk factors, and time course of sensory extinction in the acute stroke setting. Such data might help to improve our understanding of behavioural manifestations in acute stroke. They could also help generate new hypotheses for further studies on the pathophysiology of sensory extinction.

\section{Materials and methods}

\section{Study design, setting, and selection of participants}

This prospective cohort study was conducted on consecutive patients with acute stroke admitted to the Stroke Unit of Geneva University Hospital, from September 2012 to March 2014. The study procedure has been described previously (Kamtchum Tatuene et al. 2016). Briefly, exclusion criteria were past history of stroke, severe aphasia, and severe stroke (NIHSS score $>20$ ); and any documented alteration of visual, tactile, auditory, or $\operatorname{cog}$ nitive functions susceptible to interfere with the neuropsychological evaluation. For instance, patients with clearly defined hemianopia, hemianesthesia, or hemihypoesthesia were not included in this study. Patients with altered level of consciousness were not systematically excluded but rather examined later depending on their capacity to cooperate. Patients were clinically tested for sensory extinction as soon as possible after their admission (visit 1). Five subtypes of sensory extinction were considered: homologous and heterologous tactile extinction as well as visual, auditory, and auditory-tactile cross-modal extinction. To perform the clinical assessment of patients, one of us (JKT) trained and evaluated three examiners.

During visit 1 , we also recorded data on factors potentially related to sensory extinction: age, gender, handedness, stroke severity on the examination day as assessed by the National Institute of Health Stroke Scale (NIHSS) (Brott et al. 1989), presence of peripersonal visuospatial neglect (PVN), and stroke type (ischemic/hemorrhagic). We also determined lesion volume in $\mathrm{mL}$ (provided by the MRIcro software, see "Anatomical study and mapping of brain lesions") and location (right/left hemisphere or bilateral) on plain computed tomography (CT) scans (for haemorrhagic stroke) or Diffusion-Weighted-Imaging sequences (DWI) obtained with a $3 \mathrm{~T}$ Magnetic Resonance Imaging (MRI) scanner within 15 days of symptoms onset (for ischaemic stroke).

All patients diagnosed with sensory extinction on visit 1 were then systematically re-evaluated 2 (visit 2), 7 (visit 3), 15 (visit 4), 30 (visit 5), and 90 (visit 6) days later. Follow- 
up was terminated once a patient did not present any type of sensory extinction on two consecutive visits.

\section{Procedures for the neuropsychological evaluation}

During clinical assessment, patients were seated either in bed or, when possible, at a desk. They were requested to keep their eyes closed except when tested for visual extinction or when performing paper-and-pencil tasks. For each unimodal sensory extinction task (tactile, auditory or visual), stimulation sequences were established in advance and comprised ten unilateral (five ipsilesional and five contralesional) and ten bilateral simultaneous stimuli. These stimuli were randomly distributed in time (fixed random schedule) with the exception that the first one was always ipsilesional (following the common medical practice of assessing the unaffected side first). The number of correct answers for each type of stimulation was recorded. Regarding cut-off values, we could not rely on established rules from the literature as different decision thresholds have been used (Becker and Karnath 2007; Umarova et al. 2011; Vallar et al. 1994; Vossel et al. 2011). In this study, patients were classified as showing extinction if they met all the following three criteria: $100 \%$ correct answers for single ipsilesional stimuli, at least $80 \%$ correct answers for single contralesional stimuli and less than $80 \%$ correct answers for bilateral stimuli (Vallar et al. 1994). Patients showing less than $80 \%$ correct answers for single contralesional stimuli were considered as having a sensory deficit and were excluded.

Being aware that test results could be influenced by fluctuations of the strength of stimuli or asynchrony of stimulus onset and termination during bilateral stimulations, all neuropsychological tests were performed twice during each visit using non-standardized stimuli the first time and standardized stimuli the second time as described below.

\section{Testing for homologous and heterologous tactile extinction}

For these tasks, whenever possible, the examiner stood in front of the patient in the midsagittal plane or as close as possible to this plane for patients examined in bed. The tactile stimulus consisted of a brief slight touch applied by the examiner's fingertip. When testing for homologous tactile extinction (same body part stimulated on both sides), the tactile stimulus was administered to the patient's right or left cheek, or to both cheeks simultaneously. Before examination, patients were informed that stimuli could be single or double and that they were to give a verbal response ("single—right", "single—left", or "double"). When testing for heterologous tactile extinction, the stimulus was administered either to the patient's ipsilesional cheek, the dorsal surface of the patient's contralesional hand or the ipsilesional cheek, and the contralesional hand (dorsal surface), simultaneously. Before examination, patients were informed that stimuli could be single or double and that they were to give a verbal response ("single—cheek", "single—hand", or "double").

\section{Testing for visual extinction}

We used the confrontation technique (Chechlacz et al. 2014; Umarova et al. 2011). The patient was instructed to keep looking at the examiner's nose located at $60 \mathrm{~cm}$ distance in the midsagittal plane. The visual stimuli consisted of a brief movement (rapid flexion-extension) of the examiner's index finger either in the right or left visual hemifield or simultaneously in both visual hemifields. The examiner's right and left fingers were placed halfway between the examiner and the patient facing him, at $45^{\circ}$ eccentricity on the horizontal plane. Before examination, patients were informed that stimuli could be single or double and that they were to give a verbal response ("single—right", "single—left", or "double").

\section{Testing for auditory extinction}

The auditory stimuli consisted of a brief sound produced by the examiner, within $5 \mathrm{~cm}$ from the external auditory meatus, either on the right or left side or on both sides, simultaneously. This close distance was chosen, because extinction and other multimodal integration phenomena are known to be stronger in the near peripersonal space (Graziano et al. 1999; Jacobs et al. 2011). The sound was produced by snapping fingers. Before examination, patients were informed that stimuli could be single or double and that they were to give a verbal response ("sound—right", "sound-left", or "double").

\section{Testing for auditory-tactile cross-modal extinction}

We used two sets of 20 stimulations. Each set was made up of ten unimodal stimulations (auditory or tactile as defined above) randomly administered either unilaterally or bilaterally, and ten cross-modal stimulations combining an auditory stimulus in one ear and a simultaneous tactile stimulus on the contralateral cheek. We decided to administer the tactile stimulus to the cheek rather than the hand, because the previous studies on visuo-tactile extinction suggested that cross-modal extinction is stronger when closely related body parts are stimulated (Farne et al. 2005; Jacobs et al. 2011). Unimodal stimulations considered as control were randomly intermixed with crossmodal stimulations. In case of cross-modal stimulation, the auditory stimulus was administered to the ipsilesional side 
for the first set of 20 stimulations and to the contralesional side for the second.

Before examination, patients were informed that stimuli could be single unimodal or double unimodal or double cross-modal. They had to give a verbal response ("touchright", "touch-left", "touch - double", "sound—right", "sound-left", "sound-double", or "touch-sound"). The number of correct answers for each type of stimulation was recorded. Patients were classified as showing contralesional auditory-tactile cross-modal extinction if they gave less than $80 \%$ correct answers for cross-modal stimulations in the first set of 20 stimulations. They were classified as showing ipsilesional auditory-tactile crossmodal extinction if they gave less than $80 \%$ correct answers for cross-modal stimulations in the second set.

We used white noise rather than pure tones to maximise our chances of identifying cases of auditory-tactile crossmodal extinction. Previous studies have demonstrated that pure tones do not activate multimodal neurons and produce milder cross-modal extinction than white noise (Graziano et al. 1999; Ladavas et al. 2001). We decided to study auditory-tactile cross-modal extinction rather than visuotactile or audio-visual cross-modal extinctions, because auditory-tactile stimuli are easier to use in patients with acute stroke who usually feel tired and anxious. Had we chosen to study visuo-tactile extinction, we would have experienced difficulties related to positioning of patients and fatigue due to sustained fixation. Moreover, we thought that results found with auditory-tactile cross-modal extinction could be easily repeated when using other types of cross-modal extinction (Ladavas et al. 1998, 2001). Indeed, pathological findings could be aggravated due to the dominance of visual stimuli first described by Colavita in 1974 (Spence 2009).

\section{Testing for peripersonal visuospatial neglect (PVN)}

Two paper-and-pencil tasks were used: the Ota's gap detection task (Ota et al. 2001) and a line bisection task (Azouvi et al. 2006). These tests were administered and interpreted as reported previously (Kamtchum Tatuene et al. 2016).

\section{Standardization of testing procedures}

Tactile, auditory, and auditory-tactile cross-modal extinction tasks were performed twice for each patient during each visit. Non-standardized tactile (slight touch with the tip of the index) and auditory (fingers snapping) stimuli were used the first time, whereas standardized tactile and auditory stimuli were used the second time. Standardized tactile stimuli were administered with a calibrated 5.07/ $10 \mathrm{~g}$ Semmes-Weinstein monofilament (Feng et al. 2009), while standardized auditory stimuli consisted of a preregistered click-like white noise administered through a headset connected to a computer. Examiners were trained for simultaneous administration of standardized stimuli during bilateral bimodal stimulations. The test for visual extinction was not standardized in this study.

\section{Anatomical study and mapping of brain lesions}

The description of the location of brain lesions was done using region-involvement indices as reported previously (Kamtchum Tatuene et al. 2016). The following functional regions of the brain were attributed a score of 1 if they were partially or totally affected by the acute stroke or 0 if not involved at all: frontal, insular, rolandic, parietal, temporal, occipital, thalamic, caudate nucleus, putamen, pallidum, internal capsule, brain stem, and cerebellum. The region-specific score (RSS) was defined as the total number of times that a functional region had received a score of 1 after reviewing all the CT and MRI scans of patients with at least one subtype of sensory extinction. The region-involvement index (RII) was defined as the ratio of a RSS and the sum of all RSSs.

A lesion-overlap study was also performed to identify brain regions commonly damaged in patients with sensory extinction (Rousseaux et al. 2013; Saj et al. 2012; Verdon et al. 2010). Lesions identified on plain CT or DWI were manually reconstructed on a standardized brain template using the MRIcro software (http://www.mricro.com) (Karnath et al. 2011) to obtain a three-dimensional region of interest (ROI). The slices thickness was $2 \mathrm{~mm}$. The ROI was then used to build an overlap map. The volume of each ROI (in cubic centimetres or millilitres) was automatically displayed in the bottom left corner of the ROI editing panel. All the analyses with MRIcro were done by a trained neuropsychologist who was blind of patients' performance (AS).

\section{Ethical issues}

The study was approved by the Geneva University Hospital Ethical Committee for Research on Human Beings (Authorization number: CER 12-191). All patients included gave written informed consent to take part in the study. Access to patients' data and anonymized case report forms was restricted to authorized members of the research team.

\section{Statistical analysis}

Proportions of patients with a given characteristic and means for quantitative data were computed with a $95 \%$ confidence interval unless otherwise stated. To identify variables associated with sensory extinction, a 
multivariable logistic regression analysis was performed. Sensory extinction was considered as the dependant variable. The independent variables were age $(<60$ years as reference), sex (female as reference), side of lesion (right versus left or bilateral), type of stroke (haemorrhagic versus ischaemic or mixed), stroke severity (NIHSS score $<5$ as reference), lesion volume $(<2 \mathrm{~mL}$ as reference), time to first examination ( $>3$ days as reference), and the presence of PVN. The choice of the dichotomization threshold for continuous variables was guided by their performance for the diagnosis of sensory extinction (see online resource 1 ). $p$ values $<0.05$ were considered as significant. Statistical analysis was performed with the software STATA 13 (StataCorp LP, USA).

\section{Results}

\section{Patients' clinical characteristics}

A total of 73 patients were recruited (38.4\% women). Mean age was 62.3 years (95\% CI 58.8-65.7) and mean NIHSS score was 1.6 (range $0-10,95 \%$ CI 1.2-2.1). The mean time to first examination was 4.1 days (95\% CI 3.5-4.8) and $78.1 \%(57 / 73)$ of the patients were examined within 5 days post-stroke. The prevalence of PVN was $23.3 \%$ (17/ 73; 95\% CI 14.2-34.6). Patients' baseline characteristics and performance on the neuropsychological tests are summarized in Tables 1 and 2, respectively.

\section{Prevalence, risk factors, and time course of sensory extinction}

Ten patients had at least one subtype of sensory extinction yielding an overall prevalence of $13.7 \%$ (6.8-23.8). The prevalence of each subtype of sensory extinction is given in Table 3. In the multivariable logistic regression analysis, a lesion volume $\geq 2 \mathrm{~mL}$ (adjusted $\mathrm{OR}=38.88, p=0.04$ ) and presence of PVN (adjusted OR $=24.27, p=0.04$ ) were independent predictors of sensory extinction (Table 4). The insula, the putamen, and the pallidum were the brain regions most frequently involved in patients with sensory extinction, as shown in Table 5, and also in the lesion-overlap map (Fig. 1).

Sensory extinction was found in three patients at visits 2 and 3. Two of these patients had heterologous tactile extinction and a left hemisphere lesion, while the third had a right hemisphere lesion with visual extinction and ipsilesional auditory-tactile cross-modal extinction. No case of sensory extinction was found at visit 4 or beyond, which is 15 days after the first examination. The results of the neuropsychological evaluation during follow-up visits are summarized in Table 6.

\section{Results with standardization}

When using standardized testing procedures, the prevalence of sensory extinction was $8.2 \%$ (95\% CI 3.08-17.04). There was $89 \%$ agreement between non-standardized and standardized tests regarding the diagnosis of sensory extinction $(\kappa=0.44)$. At baseline, there was no case of homologous tactile extinction, three cases of heterologous tactile extinction, one case of auditory extinction, two cases of contralesional auditory-tactile cross-modal extinction, and no case of ipsilesional auditory-tactile cross-modal extinction. The agreement between non-standardized and standardized procedures was $100 \%$ for homologous tactile extinction $(\kappa=1), 96 \%$ for heterologous tactile extinction $(\kappa=0.64), 96 \%$ for contralesional auditory-tactile crossmodal extinction $(\kappa=0.40), 97.3 \%$ for ipsilesional auditory-tactile cross-modal extinction $(\kappa=0)$, and $94.5 \%$ for auditory extinction $(\kappa=0)$.

\section{Discussion}

This study was carried out to determine the prevalence, potential risk factors, and the time course of sensory extinction in acute stroke. Among the 73 patients included, $13.7 \%$ had at least one subtype of sensory extinction and all recovered completely within 15 days after the first examination. Lesion volume $\geq 2 \mathrm{~mL}$ and presence of PVN were independent predictors of sensory extinction. Given that the standardization of testing procedures did not significantly increase the diagnostic yield, the discussion will be based solely on results obtained with non-standardized procedures that are more representative of the real-life practice in the acute stroke setting.

Studies of sensory extinction and neglect in acute stroke are needed to clarify their pathophysiological relation. The major logistic challenge of our exploratory cohort study was to develop a simple and practical, yet scientifically valid evaluation of sensory extinction in the acute stroke setting where disorders of attention and language are highly prevalent. Being aware of the fact that the pathophysiology of sensory extinction may involve directional and non-directional deficits of attention (de Haan et al. 2015), the critical issue was rather to minimize the false positive rate of extinction than to completely eliminate any single attentional deficit in patients before carrying out the neuropsychological tests. We believe that the following measures helped us to reasonably achieve this goal, though at the cost of lower inclusion rates in the group of moderate and severe strokes: (1) stringent selection criteria to exclude all patients in whom reduced alertness would have been a major confounder (elderly patients with pre-existing cognitive decline, patients with NIHSS $>20$ on the 
Table 1 Baseline clinical characteristics of patients included

\begin{tabular}{|c|c|c|c|}
\hline \multirow[t]{2}{*}{ Factors studied } & \multicolumn{2}{|l|}{ Sensory extinction } & \multirow[t]{2}{*}{ Total } \\
\hline & $\mathrm{Yes}^{\mathrm{a}}$ & No & \\
\hline \multicolumn{4}{|l|}{ Sex } \\
\hline Male & $5(11.1)$ & 40 & 45 \\
\hline Female & $5(17.9)$ & 23 & 28 \\
\hline Age & $66.8(56.6-77.0)$ & $61.5(57.7-65.3)$ & $62.3(58.8-65.7)$ \\
\hline \multicolumn{4}{|l|}{ Side of lesion } \\
\hline Right & $3(8.3)$ & 33 & 36 \\
\hline Left & $7(21.2)$ & 26 & 33 \\
\hline Bilateral lesions & $0(0)$ & 4 & 4 \\
\hline \multicolumn{4}{|l|}{ Type of lesion } \\
\hline Ischemic & $9(14.1)$ & 55 & 64 \\
\hline Haemorrhagic & $1(11.1)$ & 8 & 9 \\
\hline Stroke severity (NIHSS score) ${ }^{b}$ & $2.7(0.8-4.6)$ & $1.4(1.0-1.8)$ & $1.6(1.2-2.1)$ \\
\hline Lesion volume (mL) & $17.3(0.2-34.4)$ & $6.5(3.9-9.0)$ & $8.0(4.9-11.0)$ \\
\hline Time to first examination & $4.2(3.5-4.9)$ & $4.0(3.2-4.8)$ & $4.1(3.5-4.8)$ \\
\hline \multicolumn{4}{|l|}{ Handedness } \\
\hline Right-handed & $10(15.2)$ & 56 & 66 \\
\hline Left-handed & $0(0)$ & 7 & 7 \\
\hline \multicolumn{4}{|l|}{ Visuospatial neglect } \\
\hline No & $6(11.1)$ & 50 & 56 \\
\hline Yes & $4(30.0)$ & 13 & 17 \\
\hline
\end{tabular}

${ }^{a}$ For categorical variables, the frequency is given with the percentage of total in the corresponding row. For continuous variables, the mean is given with the $95 \%$ confidence interval

b The NIHSS score ranged from 0 to 8 in patients with sensory extinction and from 0 to 10 in patients without sensory extinction examination day), (2) a small number of trained and assessed examiners to ensure consistency of results, and (3) standardized sets of stimuli with cutoffs to eliminate false positives due to either random fluctuations of patients' alertness during the assessment, random asymmetry of the strength of stimuli or random temporal asynchrony of stimulus onset, and termination during bilateral stimulations.

In this study, patients with bigger lesions were more likely to have at least one subtype of sensory extinction. This result may be explained by the fact that bigger lesions affect several functional brain regions in the human attentional network thus having a greater impact on the attentional capacity. As a consequence, the predominance of patients with small lesions in our sample suggests that the real prevalence of sensory extinction is underestimated. The independent association between sensory extinction and visuospatial neglect found in this work could be considered as an additional argument to support the hypothesis that there is some overlap in their pathophysiology. Further studies in the acute stroke setting are expected to disentangle the complex relation between these deficits. Unexpectedly, the side of the brain lesion was not significantly associated with the presence of sensory extinction. The previous studies of visual extinction have reported a higher prevalence in patients with right brain lesions, as is the case for visuospatial neglect (Becker and Karnath 2007; de Haan et al. 2012). There are three hypotheses that could explain this discrepancy.

First, it is possible that some patients with right hemisphere lesions (especially those involving the parietal lobe) and severe sensory neglect were mistakenly classified as having hemihypoesthesia or hemianesthesia upon admission and, therefore, not included in our study. These patients could, therefore, not undergo further testing for tactile, visual, or auditory extinction. Together with the stringent selection criteria, the exclusion of some patients with right hemisphere lesions could account for the low frequency of visual extinction leading to an underestimation of the overall prevalence of sensory extinction in our sample. Indeed, it is known that visual extinction, like neglect, is more common in patients with right hemisphere lesions-explaining high rates usually reported in studies focusing on patients with right brain lesions (Umarova et al. 2011; Vallar et al. 1994; Vossel et al. 2011). This is thought to result from the specialization of right 
Table 2 Summarized results of the neuropsychological tests at baseline
Behavioural disorder and evaluation measures

Sensory extinction

Yes

No

Peripersonal visuospatial neglect ${ }^{\mathrm{a}}$

Ota's gap detection task

Number of targets omitted on the left

$1.5 \pm 1.3$

$0.1 \pm 0.03$

Number of targets omitted on the right

$0.4 \pm 0.4$

$0.1 \pm 0.05$

Total number of targets omitted

$1.9 \pm 1.7$

$0.2 \pm 0.06$

Line bisection task

Rightward deviation in $\mathrm{mm}$ (5 cm line)

Leftward deviation in $\mathrm{mm}$ ( $5 \mathrm{~cm}$ line)

Rightward deviation in $\mathrm{mm}$ (20 cm line)

$0.9 \pm 0.3$

$0.9 \pm 0.1$

$1.3 \pm 0.7$

$1.4 \pm 0.3$

$2.6 \pm 0.9$

$3.4 \pm 0.5$

Leftward deviation in $\mathrm{mm}(20 \mathrm{~cm}$ line $)$

$7.9 \pm 2.4$

$2.7 \pm 0.4$

Sensory extinction ${ }^{\mathrm{b}}$

Homologous tactile extinction

Unilateral stimulation on the left

Without standardization

With standardization

$100.0 \pm 0.0$

$100 \pm 0.0$

$96.0 \pm 4.0$

$100 \pm 0.0$

Unilateral stimulation on the right

Without standardization

With standardization

$100.0 \pm 0.0$

$99.7 \pm 0.3$

$98.0 \pm 2.0$

$99.7 \pm 0.3$

Bilateral stimulations

Without standardization

With standardization

Heterologous tactile extinction

Unilateral stimulation on the left

Without standardization

With standardization

Unilateral stimulation on the right

Without standardization

With standardization

Bilateral stimulations

Without standardization

With standardization

Visual extinction

Unilateral stimulation on the left

Without standardization

Unilateral stimulation on the right

Without standardization

$98.0 \pm 2.0$

$99.8 \pm 0.2$

$99 \pm 1.0$

$100 \pm 0.0$

$100.0 \pm 0.0$

$99.7 \pm 0.3$

$90.0 \pm 10.0$

$100 \pm 0.0$

$100.0 \pm 0.0$

$99.4 \pm 0.4$

$90.0 \pm 10.0$

$99.7 \pm 0.3$

$60.0 \pm 13.0$

$98.4 \pm 0.6$

$69.0 \pm 13.4$

$96.8 \pm 0.8$

Bilateral stimulations

Without standardization

Auditory extinction

Unilateral stimulation on the left

Without standardization

With standardization

Unilateral stimulation on the right

Without standardization

With standardization

$100.0 \pm 0.0$

$100.0 \pm 0.0$

$100.0 \pm 0.0$

$99.7 \pm 0.3$

$94.0 \pm 4.0$

$99.7 \pm 0.2$

Bilateral stimulations

Without standardization

With standardization

$\begin{array}{rr}100.0 & \pm 0.0 \\ 100.0 & \pm 0.0 \\ & 100.0 \pm 0.0 \\ 98.0 & \pm 2.0 \\ 100.0 & \pm 0.0 \\ & 100.7 \pm 0.3 \\ 84.0 & \pm 0.0 \\ 99 & \pm 1.0 \\ \end{array}$


Table 2 continued

Table 3 Prevalence of various subtypes of sensory extinction

\begin{tabular}{lll}
\hline Behavioural disorder and evaluation measures & \multicolumn{2}{l}{ Sensory extinction } \\
\cline { 3 - 3 } & Yes & No \\
\hline Contralesional auditory-tactile cross-modal extinction & & $97.3 \pm 0.7$ \\
$\quad$ Without standardization & $74.0 \pm 10.6$ & $99.0 \pm 0.6$ \\
$\quad$ With standardization & $94.0 \pm 5.0$ & \\
Ipsilesional auditory-tactile cross-modal extinction & & $99.2 \pm 0.4$ \\
$\quad$ Without standardization & $91.0 \pm 5.5$ & $99.4 \pm 0.3$ \\
$\quad$ With standardization & $97.0 \pm 2.1$ & \\
\hline
\end{tabular}

${ }^{a}$ For peripersonal visuospatial neglect, the mean number of targets omitted or the mean deviation is presented with the standard error

${ }^{b}$ For sensory extinction, the mean percentage of correct detection for each type of stimulation is presented with the standard error

\begin{tabular}{llll}
\hline Extinction type & Presence of visuospatial neglect & Count & $\begin{array}{l}\text { Total } \\
n(\%, \mathrm{CI})^{\mathrm{a}}\end{array}$ \\
\hline Tactile & & & \\
Homologous & - & 0 & $6(8.2 \%, 3.1-17.0)$ \\
Heterologous & Yes & 2 & \\
No & No & $4^{\mathrm{b}}$ & $3(4.1 \%, 0.9-11.5)$ \\
Visual & Yes & 3 & $1(1.4 \%, 0-7.4)$ \\
Cross-modal (auditory-tactile) & 1 & $4(5.5 \%, 1.5-13.4)$ \\
Ipsilesional & No & & \\
Contralesional & No & 1 & \\
Bilateral & Yes & 2 & \\
\hline
\end{tabular}

${ }^{a}$ Estimated prevalence and confidence interval

b Among patients with heterologous tactile extinction, one also had auditory extinction and two had auditory-tactile cross-modal extinction

c The patient with bilateral auditory-tactile cross-modal extinction also had visual extinction

Table 4 Univariable and multivariable analyses of factors associated with sensory extinction

\begin{tabular}{|c|c|c|c|c|c|c|}
\hline \multirow[t]{2}{*}{ Characteristics $^{\mathrm{a}}$} & \multicolumn{3}{|c|}{ Univariable model } & \multicolumn{3}{|c|}{ Multivariable model } \\
\hline & Crude OR & $95 \% \mathrm{CI}$ & $p$ & Adjusted OR & $95 \% \mathrm{CI}$ & $p$ \\
\hline Male & 0.58 & $0.15-2.20$ & 0.42 & 1.77 & $0.27-11.41$ & 0.55 \\
\hline Age $\geq 60$ years & 3.2 & $0.63-16.29$ & 0.16 & 8.33 & $0.68-101.30$ & 0.10 \\
\hline Right hemisphere lesion & 0.39 & $0.09-1.64$ & 0.20 & 0.27 & $0.04-2.04$ & 0.21 \\
\hline Haemorrhagic stroke & 0.76 & $0.09-6.86$ & 0.81 & 0.46 & $0.03-7.56$ & 0.60 \\
\hline NIHSS score $\geq 5$ & 13.07 & $1.85-92.12$ & 0.01 & 17.51 & $0.67-458.84$ & 0.08 \\
\hline Lesion volume $\geq 2 \mathrm{~mL}$ & 2.46 & $0.48-12.57$ & 0.28 & 38.88 & $1.21-1245.17$ & 0.04 \\
\hline Time to first examination $\leq 3$ days & 0.83 & $0.21-3.24$ & 0.79 & 0.62 & $0.10-3.65$ & 0.60 \\
\hline \multirow[t]{2}{*}{ Visuospatial neglect } & 2.56 & $0.63-10.45$ & 0.19 & 24.27 & $1.13-519.93$ & 0.04 \\
\hline & & & & \multicolumn{3}{|c|}{$\begin{array}{l}\text { Area under the } \mathrm{ROC} \text { curve }=0.90 \\
R^{2}=0.864\end{array}$} \\
\hline
\end{tabular}

\footnotetext{
${ }^{a}$ The handedness was not included in the model, because all the patients with sensory extinction are right-handed
} 
Table 5 Overview of the functional brain regions damaged in patients with at least one subtype of sensory extinction $(n=10)$

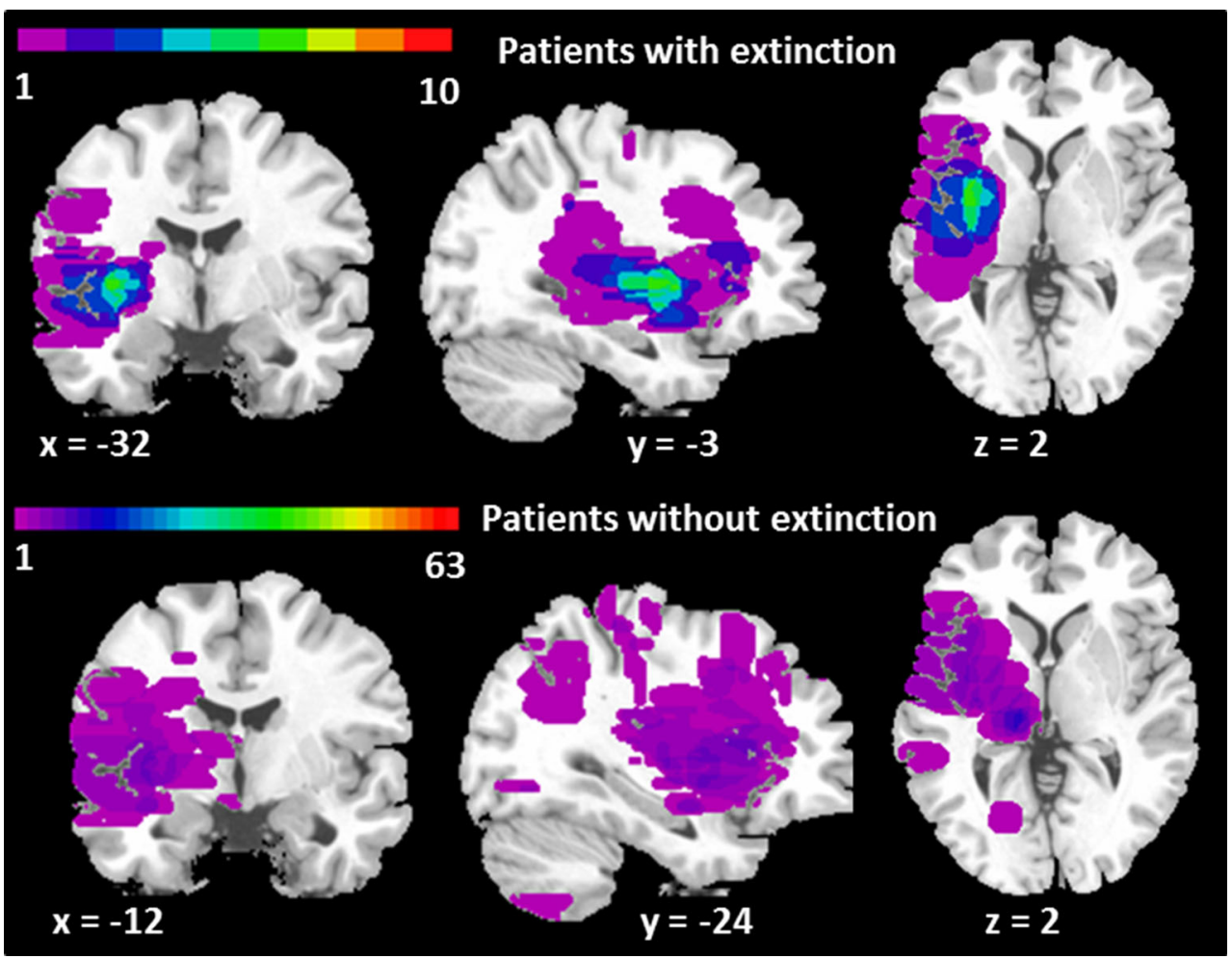

\begin{tabular}{llllllllllllll}
\hline Side of lesion $^{\mathrm{a}}$ & Fr & Ins & Rol & Par & Temp & Occ & Thal & CN & Put & Pal & IC & BS & C \\
\hline RSS (left) & 1 & 4 & 2 & 3 & 3 & 0 & 0 & 1 & 3 & 4 & 3 & 1 & 0 \\
RSS (right) & 1 & 1 & 2 & 0 & 1 & 0 & 0 & 0 & 2 & 1 & 0 & 0 & 0 \\
Global RSS & 2 & 5 & 4 & 3 & 4 & 0 & 0 & 1 & 5 & 5 & 3 & 1 & 0 \\
Global RII & 0.06 & 0.15 & 0.12 & 0.09 & 0.12 & 0.00 & 0.00 & 0.03 & 0.15 & 0.15 & 0.09 & 0.03 & 0.00 \\
\hline
\end{tabular}

$H$ handedness ( $R$ right-handed, $L$ left-handed), $F r$ frontal, Ins insular, Rol rolandic, Par parietal, Temp temporal, Occ occipital, Thal thalamus, $C N$ caudate nucleus, Put putamen, Pall pallidum, IC internal capsule, $B S$ brainstem, $C$ cerebellum, $R S S$ region-specific score, $R I I$ region-involvement index

a There were seven patients with left hemisphere lesion and three patients with right hemisphere lesion
Fig. 1 Lesion-overlap map for patients with and without sensory extinction. Lesions on the right side have been flipped to the left side to facilitate the global analysis. The coordinates $(x, y$, and $z)$ of the region of maximum overlap are given in the Talairach's 3D space. Colour codes represent the number of patients with damage to a given area, ranging from purple for areas affected in one patient only, to red for areas affected in all patients. In patients with sensory extinction, the region of maximum overlap (green) is affected in six patients (out of ten) and corresponds to the insular, putaminal, and the pallidal functional regions that had the highest region-involvement indices (Table 5) 
Table 6 Neuropsychological outcome of patients with sensory extinction at visit 1

\begin{tabular}{|c|c|c|c|c|c|c|c|c|c|c|c|}
\hline \multirow{2}{*}{$\begin{array}{l}\text { Patient } \\
\text { ID }\end{array}$} & \multirow{2}{*}{$\begin{array}{l}\text { Side of } \\
\text { lesion }\end{array}$} & \multirow{2}{*}{$\begin{array}{l}\text { Stroke severity } \\
\text { (NIHSS score) }\end{array}$} & \multirow{2}{*}{$\begin{array}{l}\text { Lesion volume } \\
(\mathrm{mL})\end{array}$} & \multicolumn{2}{|l|}{ Visit 1} & \multicolumn{2}{|l|}{ Visit 2} & \multicolumn{2}{|l|}{ Visit 3} & \multicolumn{2}{|l|}{ Visit 4} \\
\hline & & & & Extinction & PVN & Extinction & PVN & Extinction & PVN & Extinction & PVN \\
\hline 18 & Right & 0 & 2.4 & Yes & No & No & No & No & No & No & No \\
\hline $37^{\mathrm{a}}$ & Right & 1 & 68.8 & Yes & Yes & Yes & Yes & Yes & Yes & No & No \\
\hline 43 & Right & 2 & 2.1 & Yes & No & No & No & No & No & No & No \\
\hline 101 & Left & 6 & 1.9 & Yes & Yes & No & Yes & No & No & No & No \\
\hline $30^{\mathrm{a}}$ & Left & 8 & 50.7 & Yes & No & Yes & No & Yes & No & No & No \\
\hline 50 & Left & 2 & 1.2 & Yes & Yes & No & No & No & No & No & No \\
\hline $22^{\mathrm{a}}$ & Left & 1 & 5.1 & Yes & No & Yes & No & Yes & No & No & No \\
\hline 6 & Left & 1 & 24.3 & Yes & No & No & No & No & No & No & No \\
\hline 87 & Left & 5 & 3.7 & Yes & Yes & No & Yes & No & Yes & No & No \\
\hline 25 & Left & 1 & 12.9 & Yes & No & No & No & No & No & No & No \\
\hline
\end{tabular}

${ }^{a}$ Patients with sensory extinction at visits 2 and 3

could be involved in this rapid recovery: (1) restoration of perfusion to penumbral regions rendered temporarily nonfunctional but not permanently injured by moderate degrees of ischemia, (2) resolution of cytotoxic oedema responsible for compression of tissues surrounding the infarct, (3) unmasking of redundant underused neural pathways, and (4) the early neural repair and network reorganization (Dobkin 1996).

This study has several strengths: the early recruitment of patients with acute stroke, use of repeated testing that increases the reliability of results, assessment of multiple subtypes of sensory extinction that increases the sensitivity of the screening, and longitudinal follow-up of patients with sensory extinction that allowed us to report the duration of this symptom for the first time. The lack of a voxel-based lesion statistical mapping (VLSM) analysis (Saj et al. 2012) is a major limitation of this study. Such analysis would have helped to refine our understanding of the relation between sensory extinction and the location of acute brain lesions. However, the validity of a post hoc analysis of the neuroanatomical correlates of sensory extinction would have been questionable given the low prevalence of sensory extinction in our sample. Moreover, such analysis would rely on the assumption that all subtypes of sensory extinction have the same anatomical substrate which would be highly speculative. A second limitation is the absence of a measure of interrater agreement for the neuropsychological tests. However, given that this study was carried out in the acute stroke setting with patients under physical and emotional stresses due to the diagnosis, the treatment, and the multiple paraclinical examinations and clinical trials going on at the same time, it was neither practically feasible nor ethically acceptable to have the same examinations performed by all three examiners at the same time for each visit. A third limitation is the lack of information on the neuropsychological rehabilitation programmes that could have influenced the time course of extinction in our cohort. Nevertheless, given that most of our patients had a mild stroke, it is unlikely that they have received specific rehabilitation therapies that could significantly interfere with our results. Indeed, all patients benefited for the standard stroke management protocol at the Geneva stroke unit. In this protocol, a specific neuropsychological rehabilitation programme is implemented only if it is deemed indispensable for the recovery. Other limitations are the small sample size and the heterogeneity in the time to the first examination. Nearly, 22\% of our patients have had their first examination beyond the fifth day after stroke onset and it is not possible to know if they had sensory extinction in the early days that had been missed leading to underestimation of the overall prevalence. The delay is explained by various factors related to the acute stroke setting: time to obtain the informed consent, availability of the patients and the examiners, interference with the clinical management of the patient, and temporary stay in the intensive care unit before transfer into the stroke unit.

In conclusion, our study shows that sensory extinction is a rare and transient phenomenon in patients with mild acute stroke. Our results also indicate that the presence of PVN and lesion volume greater than $2 \mathrm{~mL}$ are independent predictors of sensory extinction. Accurately, determining the prevalence of sensory extinction in the acute stroke setting is difficult because of the concomitant presence of disorders of alertness or non-spatial attention and the low inclusion rates due to clinical status and higher tendency to withhold consent. As these results were obtained in a small sample with most patients having a low NIHSS score, they need to be confirmed in a larger cohort using more inclusive selection criteria adapted to the clinical context and 
the specific subtype of extinction investigated to avoid selection bias. The following questions could also be addressed in upcoming studies: (1) Do all subtypes of extinction have the same neural correlates? (2) Does extinction appear in patients with severe visuospatial neglect as they recover, suggesting that there is an overlap in their pathophysiology?

Acknowledgements We express our gratitude to: (1) all the patients for kindly accepting to take part in this study; (2) our clinical research assistants Virginie FAVRE (Stroke Nurse) and Irène FREY (Neuropsychologist); and (3) Prof. Thomas PERNEGER (Division of Clinical Epidemiology) who provided valuable advices for the data analysis.

\section{Compliance with ethical standards}

\section{Conflict of interest None.}

Source of funding This project was funded by the Geneva University Hospital Fund for Research and Development (Project Number: PRD 1-2013-I). The funder had no role in study design, data collection and analysis, decision to publish, or preparation of the manuscript.

Open Access This article is distributed under the terms of the Creative Commons Attribution 4.0 International License (http://crea tivecommons.org/licenses/by/4.0/), which permits unrestricted use, distribution, and reproduction in any medium, provided you give appropriate credit to the original author(s) and the source, provide a link to the Creative Commons license, and indicate if changes were made.

\section{References}

Azouvi P, Bartolomeo P, Beis JM, Perennou D, Pradat-Diehl P, Rousseaux M (2006) A battery of tests for the quantitative assessment of unilateral neglect. Restor Neurol Neurosci 24(4-6):273-285

Becker E, Karnath HO (2007) Incidence of visual extinction after left versus right hemisphere stroke. Stroke 38(12):3172-3174

Bender MB (1952) Disorders in perception; with particular reference to the phenomena of extinction and displacement, American Lecture Series. Thomas, Springfield

Brott T, Adams HP Jr, Olinger CP, Marler JR, Barsan WG, Biller J et al (1989) Measurements of acute cerebral infarction: a clinical examination scale. Stroke 20(7):864-870

Chechlacz M, Rotshtein P, Demeyere N, Bickerton WL, Humphreys GW (2014) The frequency and severity of extinction after stroke affecting different vascular territories. Neuropsychologia 54:11-17

Cocchini G, Cubelli R, Della Sala S, Beschin N (1999) Neglect without extinction. Cortex 35(3):285-313

de Haan B, Karnath HO, Driver J (2012) Mechanisms and anatomy of unilateral extinction after brain injury. Neuropsychologia 50(6): 1045-1053

de Haan B, Stoll T, Karnath HO (2015) Early sensory processing in right hemispheric stroke patients with and without extinction. Neuropsychologia 73:141-150

Di Pellegrino G, De Renzi E (1995) An experimental investigation on the nature of extinction. Neuropsychologia 33(2):153-170
Dobkin BH (1996) Neurologic rehabilitation. FA Davis, Philadelphia

Driver J, Vuilleumier P (2001) Perceptual awareness and its loss in unilateral neglect and extinction. Cognition 79(1-2):39-88

Farne A, Dematte ML, Ladavas E (2005) Neuropsychological evidence of modular organization of the near peripersonal space. Neurology 65(11):1754-1758

Feng Y, Schlosser FJ, Sumpio BE (2009) The Semmes Weinstein monofilament examination as a screening tool for diabetic peripheral neuropathy. J Vasc Surg 50(3):675-682

Graziano MS, Reiss LA, Gross CG (1999) A neuronal representation of the location of nearby sounds. Nature 397(6718):428-430

Heilman KM, Valenstein E (2012) Clinical neuropsychology, 5th edn. Oxford University Press, Oxford

Heilman KM, Watson RT, Valenstein E (2012) Neglect and related disorders. In: Heilman KM, Valenstein E (eds) Clinical neuropsychology. Oxford University Press, Oxford, pp 296-348

Humphreys GW, Riddoch MJ, Nys G, Heinke D (2002) Transient binding by time: neuropsychological evidence from anti-extinction. Cogn Neuropsychol 19(4):361-380

Jacobs S, Brozzoli C, Hadj-Bouziane F, Meunier M, Farne A (2011) Studying multisensory processing and its role in the representation of space through pathological and physiological crossmodal extinction. Front Psychol 2:89

Kamtchum Tatuene J, Allali G, Saj A, Bernati T, Sztajzel R, Pollak P et al (2016) Incidence, risk factors and anatomy of peripersonal visuospatial neglect in acute stroke. Eur Neurol 75(3-4):157-163

Karnath HO (1988) Deficits of attention in acute and recovered visual hemi-neglect. Neuropsychologia 26(1):27-43

Karnath HO, Rorden C (2012) The anatomy of spatial neglect. Neuropsychologia 50(6):1010-1017

Karnath HO, Himmelbach M, Kuker W (2003) The cortical substrate of visual extinction. NeuroReport 14(3):437-442

Karnath HO, Rennig J, Johannsen L, Rorden C (2011) The anatomy underlying acute versus chronic spatial neglect: a longitudinal study. Brain 134(Pt 3):903-912

Kinsbourne M (1977) Hemi-neglect and hemisphere rivalry. Adv Neurol 18:41-49

Ladavas E, Zeloni G, Farne A (1998) Visual peripersonal space centred on the face in humans. Brain 121(Pt 12):2317-2326

Ladavas E, Pavani F, Farne A (2001) Auditory peripersonal space in humans: a case of auditory-tactile extinction. Neurocase 7(2):97-103

Liu CC, Veldhuijzen DS, Ohara S, Winberry J, Greenspan JD, Lenz FA (2011) Spatial attention to thermal pain stimuli in subjects with visual spatial hemi-neglect: extinction, mislocalization and misidentification of stimulus modality. Pain 152(3):498-506

Mesulam MM (1981) A cortical network for directed attention and unilateral neglect. Ann Neurol 10(4):309-325

Ota H, Fujii T, Suzuki K, Fukatsu R, Yamadori A (2001) Dissociation of body-centered and stimulus-centered representations in unilateral neglect. Neurology 57(11):2064-2069

Priftis K, Bonato M, Zorzi M, Umilta C (2013) Spatial and nonspatial aspects of neglect. Front Hum Neurosci 7:25

Punt TD, Riddoch MJ, Humphreys GW (2013) Motor extinction: a deficit of attention or intention? Front Hum Neurosci 7:644

Riddoch MJ, Rappaport SJ, Humphreys GW (2009) Extinction: a window into attentional competition. Prog Brain Res 176:149-159

Rorden C, Jelsone L, Simon-Dack S, Baylis LL, Baylis GC (2009) Visual extinction: the effect of temporal and spatial bias. Neuropsychologia 47(2):321-329

Rousseaux M, Honore J, Vuilleumier P, Saj A (2013) Neuroanatomy of space, body, and posture perception in patients with right hemisphere stroke. Neurology 81(15):1291-1297 
Saj A, Vuilleumier P (2013) Hemispatial neglect. In: Godefroy O (ed) Behavioral neurology in acute stroke management. Cambridge University Press, Cambridge, pp 126-157

Saj A, Verdon V, Vocat R, Vuilleumier P (2012) The anatomy underlying acute versus chronic spatial neglect' also depends on clinical tests. Brain 135(Pt 2):e207 (author reply e208)

Spence C (2009) Explaining the Colavita visual dominance effect. Prog Brain Res 176:245-258

Umarova RM, Saur D, Kaller CP, Vry MS, Glauche V, Mader I et al (2011) Acute visual neglect and extinction: distinct functional state of the visuospatial attention system. Brain $134(\mathrm{Pt}$ 11):3310-3325

Vallar G, Rusconi ML, Bignamini L, Geminiani G, Perani D (1994) Anatomical correlates of visual and tactile extinction in humans: a clinical CT scan study. J Neurol Neurosurg Psychiatry 57(4):464-470

Verdon V, Schwartz S, Lovblad KO, Hauert CA, Vuilleumier P (2010) Neuroanatomy of hemispatial neglect and its functional components: a study using voxel-based lesion-symptom mapping. Brain 133(Pt 3):880-894
Vossel S, Eschenbeck P, Weiss PH, Weidner R, Saliger J, Karbe H et al (2011) Visual extinction in relation to visuospatial neglect after right-hemispheric stroke: quantitative assessment and statistical lesion-symptom mapping. J Neurol Neurosurg Psychiatry $82(8): 862-868$

Vuilleumier P (2013) Mapping the functional neuroanatomy of spatial neglect and human parietal lobe functions: progress and challenges. Ann N Y Acad Sci 1296:50-74

Vuilleumier PO, Rafal RD (2000) A systematic study of visual extinction. Between- and within-field deficits of attention in hemispatial neglect. Brain 123(Pt 6):1263-1279

Watling R, Danckert J, Linnell KJ, Cocchini G (2013) Extinction and anti-extinction: the "attentional waiting" hypothesis. Neuropsychology 27(2):275-279

Weintraub S, Mesulam MM (1987) Right cerebral dominance in spatial attention. Further evidence based on ipsilateral neglect. Arch Neurol 44(6):621-625 